\title{
Management of incidental pulmonary embolism
}

\author{
Frederikus A. Klok and Menno V. Huisman \\ Affiliation: Department of Thrombosis and Hemostasis, Leiden University Medical Center, Leiden, The \\ Netherlands. \\ Correspondence: F.A. Klok, Department of Thrombosis and Hemostasis, LUMC (C7Q-14), Albinusdreef 2 , \\ Postbus 9600, 2300 RC Leiden, The Netherlands. E-mail: f.a.klokaLUMC.nl
}

@ERSpublications

Update in diagnosis and treatment of incidental pulmonary embolism http://ow.ly/qyIb30bRwvE

Cite this article as: Klok FA, Huisman MV. Management of incidental pulmonary embolism. Eur Respir J 2017; 49: 1700275 [https://doi.org/10.1183/13993003.00275-2017].

\begin{abstract}
Incidental pulmonary embolism (PE) is a frequent finding on routine computed tomography (CT) scans of the chest, occurring in $1.1 \%$ of coronary CT scans and $3.6 \%$ of oncological CT scans. Despite this high frequency, optimal management of incidental PE has not been addressed in clinical trials and remains the subject of debate. Although these CT scans have not been performed with a dedicated PE protocol and have suboptimal contrast enhancement, diagnosis of incidental PE has been shown to be accurate up to the segmental and subsegmental arteries. The embolic load in incidental PE is lower than that in symptomatic PE. Even so, observational studies suggest that the natural course of incidental PE is similar to that of symptomatic PE with regard to the risk of recurrent venous thrombotic disease and mortality. Interestingly, the increased use of more advanced CT technology has coincided with an increase in the rate of incidental subsegmental PE, as is the case for symptomatic subsegmental PE. Although clinical trials are lacking, and observational data are limited to cancer-associated incidental PE, the consensus is that the management of incidental PE is identical to that of symptomatic PE, including the choice of optimal drug class, outpatient treatment and total duration of treatment.
\end{abstract}




\section{Introduction}

In recent years, physicians have been increasingly confronted with unsuspected filling defects of the pulmonary artery - corresponding with pulmonary embolism (PE) - identified on routine computed tomography (CT) scans of the chest, performed for indications other than suspected PE [1]. Although the available literature focusses mostly on this apparent diagnosis of incidental PE in cancer patients, this phenomenon is clearly relevant to other patients as well, including those referred for coronary artery imaging, evaluation of pulmonary infections, or those who have been subjected to major trauma. In general, unsuspected venous thrombosis accounts for an annual estimated 375000 to 425000 recognised incident cases in the US, at a total cost of $\$ 7$ billion to $\$ 10$ billion [2]. Despite this relatively high frequency, the optimal management of incidental PE has not been addressed in clinical trials and remains the subject of debate $[1,3,4]$. Here, we review the literature on this clinical entity and provide a clinical point of view for the treatment of these patients, as illustrated by a typical clinical case.

\section{Clinical case}

A 56-year old female patient was referred to the emergency ward following severe trauma, as a result of falling from her bike and crashing into a concrete wall. After a primary survey, a CT scan was performed to evaluate the extent of the injury. The CT showed multiple costal fractures, pneumothorax and atelectasis of the right lung. In addition, a subsegmental PE was found (figure 1). The patient exhibits hemodynamic and respiratory stability, with blood pressure of $156 / 92 \mathrm{mmHg}$, pulse rate of $86 / \mathrm{min}$, respiratory rate of $16 / \mathrm{min}$ and $100 \%$ saturation without the administration of oxygen. The attending trauma surgeon hesitates to start anticoagulant therapy in this patient because he fears bleeding complications.

\section{Observations}

\section{The story of 'silent' PE}

Reports on the presence of $\mathrm{PE}$ in patients with deep vein thrombosis (DVT) without respiratory symptoms, the frequency of unsuspected PE detected at autopsy, as well as notable findings from studies evaluating PE screening in high risk patients all contributed to the recognition of 'silent' PE as a relevant disease entity in the 1980s.

Several studies have investigated the frequency of scintigraphically documented PE in patients with proven symptomatic DVT, who showed no specific symptoms for PE. This incidence was reported to range from $30 \%$ to $60 \%$ [5-8]. Although these studies applied diagnostic criteria for PE other than the current standard, more recent studies that have applied the Prospective Investigation of Pulmonary Embolism Diagnosis (PIOPED) criteria or used CT pulmonary angiography (CTPA), have confirmed this high frequency (pooled weighted incidence 32\%) [9]. 'Silent PE', i.e. PE in patients with confirmed DVT, but without clear respiratory symptoms, was found to be more frequent in proximal DVT than in calf-vein thrombosis, and more frequent in unprovoked DVT than in provoked DVT $[9,10]$. Notably, the presence of 'silent $\mathrm{PE}$ ' is suggested to be associated with a higher risk of recurrence of early venous thromboembolism (VTE) during anticoagulant treatment [11, 12].

In a general hospital population, PE might be observed at autopsy in $11 \%$ to $16 \%$ of adults, a diagnosis that would have been considered ante mortem in only $3 \%$ to $31 \%$ of cases [13-16]. Observation of PE was even noted in $24 \%$ of autopsies in surgical patients [17]. An estimated $37 \%$ to $80 \%$ of these pulmonary
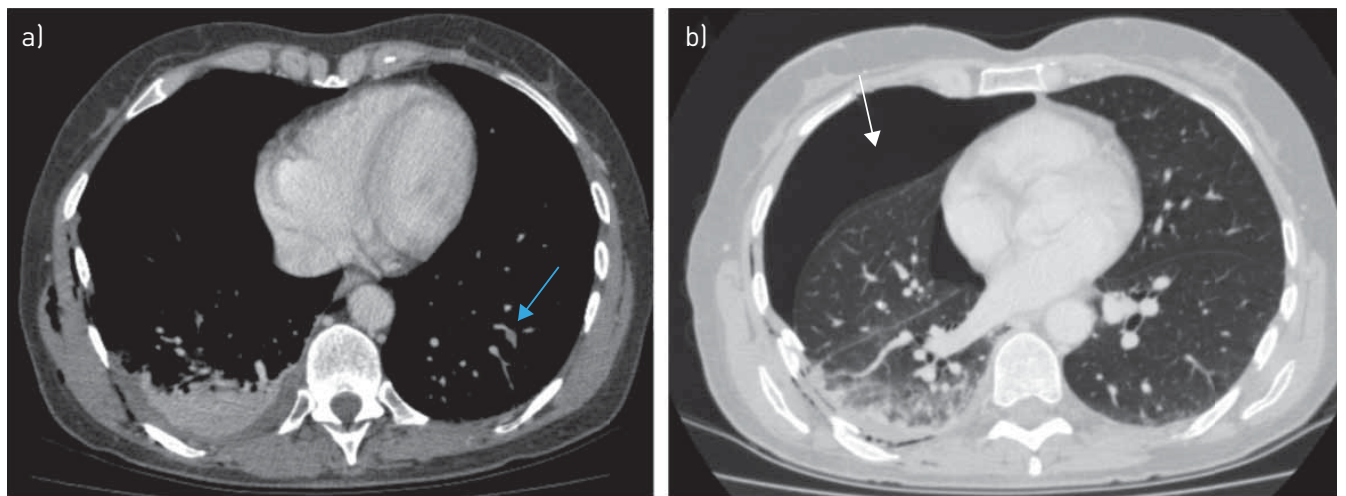

FIGURE 1 Chest computed tomography (CT) scan of patient from the clinical case. The blue arrow in panel (a) shows a filling defect in a left subsegmental pulmonary artery. Panel (b) demonstrates the pneumothorax (white arrow) and multiple costal fractures on the right side. 
emboli actually caused or significantly contributed to death, underlying the relevance of this finding. Few studies have investigated the prevalence of $\mathrm{PE}$ among high-risk patients, by performing routine ventilation/ perfusion scintigrams in patients following major orthopaedic surgery. High probability scintigram results indicative of PE were obtained in $15 \%$ to $28 \%$ of all patients, of whom less than $10 \%$ reported respiratory or chest symptoms [18-20].

Owing to the rapidly increasing use of CT scans in recent years, the clinical perspective on 'silent PE' has shifted from screening for asymptomatic PE in high-risk populations, to dealing with the clinical dilemma of incidental CT findings of uncertain clinical significance, commonly referred to as 'incidental PE' [21, 22].

\section{Frequency of incidental PE}

The reported frequency of incidental PE on routine CT-imaging of the chest varies among different patient categories, and has increased over time with the introduction of more advanced multi-slice CT machines. Generally, incidental PE is most often observed in CT scans performed for the evaluation of acute pulmonary disease and cancer staging [23]. In cancer patients, the only study available that has assessed incidental PE in 385 chest-CT scans using a 4-row CT scanner reported an incidence frequency of $2.6 \%$ (95\% confidence interval [CI] 1.2-4.7) [24]. Pooled analyses of eight studies covering 8491 cancer patients suggest that the frequency is slightly higher $(3.6 \%, 95 \%$ CI 2.6-4.6) when using modern multi-slice CT scan machines (figure 2) [25-31]. This difference is more notable when comparing the older CT-scans with modern CT-scans in a more general population referred for chest CT, with frequencies of $1.8 \%$ (3 studies, 3253 patients; 95\% CI 00.64-2.9) and 3.3\% (3 studies, 3465 patients; 95\% CI 1.6-5.0), respectively (figure 2) [32-37]. Notably, most patients in these cohorts were referred for either cancer diagnosis or follow-up as well. One study focussed on the frequency of incidental PE detected on multi-slice chest CT in trauma patients, and reported a notable 24\% (95\% CI 16-35) showing PE [38]. Furthermore, the pooled weighted frequency of incidental PE on multi-slice coronary CT in a total of 7019 patients from four studies is $1.1 \%$ (95\% CI 0.68-1.6) (figure 2) [39-42]. This lower prevalence in cardiology patients is likely caused by the fact that both active cancer and trauma are major risk factors for $\mathrm{PE}$, in contrast to coronary artery disease. The fact that patients with metastatic cancer have a higher risk of incidental PE than those with limited cancer supports this hypothesis [27, 30, 43, 44].

The reported prevalence of symptomatic subsegmental PE has increased with improvement in CT technology, and specifically the increase in the number and quality of detectors, with $4.7 \%$ of $\mathrm{PE}$ diagnosed using single-detector CT and $9.4 \%$ of patients with PE diagnosed by multi-detector CTPA [45]. The same trend is applicable to incidental subsegmental PE. Subsegmental PE was not observed in any of the 60 incidental PE diagnoses on CT scan with 4-row technology or less, (pooled frequency $0 \%$,

FIGURE 2 Weighted pooled frequencies of incidental pulmonary embolism (PE) identified on routine chest computed tomography (CT) for different patient categories and CT machines with four rows or less versus those with greater than four rows. Black bars represent point estimates with $95 \%$ confidence intervals, circles represent point estimates of individual studies; circle diameter is adapted for study size.

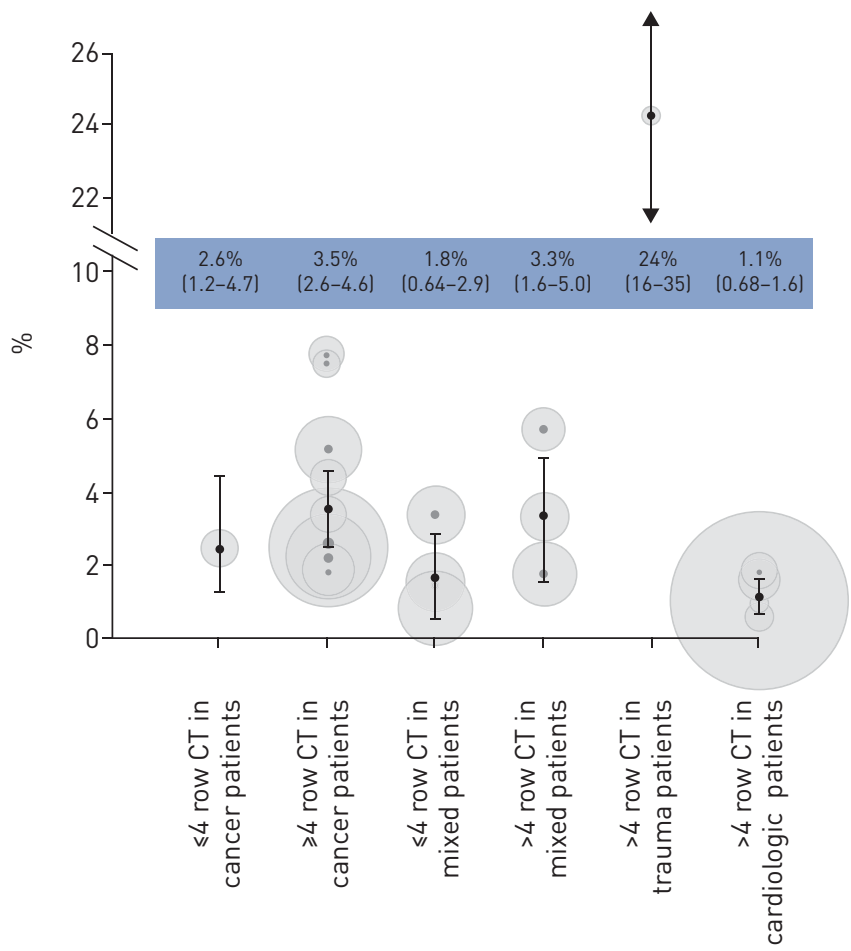


95\% CI 0-6.0) [24, 34-36], whereas 34 of the $173 \mathrm{PE}$ diagnoses on multi-row-detector CT were limited to the subsegmental pulmonary arteries (pooled weighted frequency 23\%, 95\% CI 6.7-40; figure 3) [28, 31-33, 38]. Notably, on average, the total embolic burden in incidental PE is lower than that in symptomatic PE $[23,43,46]$. In one study, the median Qanadli score index was $16 \%$ lower in 48 patients with incidental $\mathrm{PE}$, compared to a reference cohort of 113 patients with symptomatic PE, even with $40 \%$ of all incidental PEs located in the main pulmonary arteries [46-48].

Importantly, despite the frequent occurrence of subsegmental incidental PE and the overall lower embolic burden in incidental PE, interobserver agreement for incidental PE is high, as demonstrated by two studies. In the first study, two expert readers disagreed in only two of $81 \mathrm{CT}$ scans, of which 62 had previously shown incidental PE and 19 did not, for a kappa statistic of 0.93 [49]. After final consensus reading, it was concluded that the CT images of all 62 patients initially diagnosed with incidental PE were indeed positive for PE. In the second study, all 96 cases of incidental PE, diagnosed on routine CT examination were confirmed by independent review of an expert reader, for a kappa statistic of 1.0 [50]. Notably, the accuracy of a diagnosis of symptomatic isolated subsegmental PE has been questioned in a retrospective study, in which only $51 \%$ of all diagnoses were confirmed after post-hoc review of the CTPA images by an experienced chest radiologist [51]. When extrapolated to incidental PE, a reasonable recommendation would be to have the diagnosis of incidental subsegmental PE confirmed by a radiologist with specific experience [1].

In summary, incidental PE can be found in $1.1 \%$ of all coronary CT scans and $3.6 \%$ of cancer staging CT scans, a prevalence that has increased over the past years with advancing CT-technology. In particular, subsegmental incidental PE has been observed more frequently with the increased use of more advanced CT scanners.

\section{How 'silent' is incidental PE?}

Although by definition, incidental PE is an unexpected finding, upon closer scrutiny of their complaints, most patients report symptoms that are commonly associated with acute PE. In a retrospective chart review of 46 patients with incidental PE and 92 matched controls, $75 \%$ of these patients reported at least one symptom that might be associated with acute PE [52]. The patients with incidental PE were significantly more likely than control patients to complain of fatigue (54\% versus 20\%) and shortness of

FIGURE 3 Weighted pooled frequencies of subsegmental incidental pulmonary embolism (PE) on routine chest computed tomography (CT) for CT machines with four rows or less versus those with more than four rows. Black bars represent point estimates with $95 \%$ confidence intervals, circles represent point estimates of individual studies; circle diameter is adapted for study size.

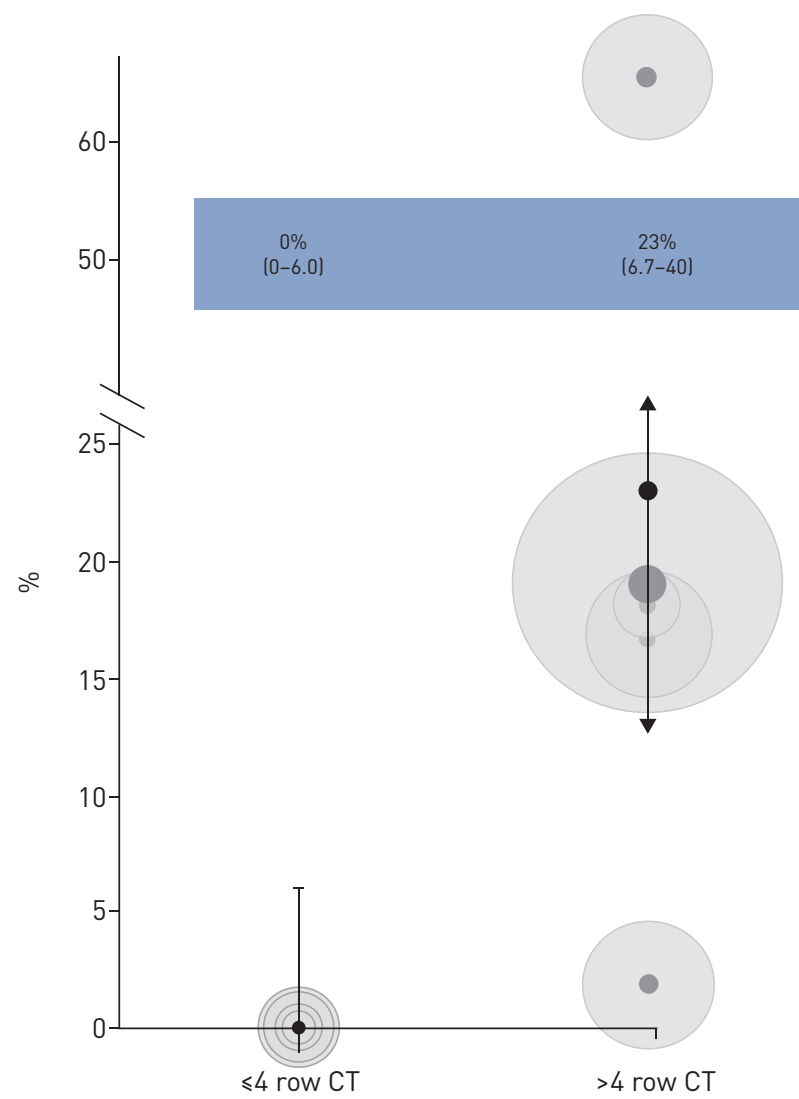


breath $(22 \%$ versus $8 \%)$, although the prevalence of tachycardia, chest pain or symptoms of DVT showed no difference. In a second publication by the same authors, after expanding the number of cases to 70 , the previous findings were confirmed and cough was added to the list of symptoms associated with incidental PE (16\% versus 5\%) [50]. The estimated frequency of PE-specific symptoms in incidental PE was lower in a third cohort (17\%) [53]. Even if recall bias and other confounding factors have influenced the findings of studies on the clinical manifestation of incidental PE, these numbers underline the fact that 'incidental' and 'silent' or 'asymptomatic' are not interchangeable terms, and the term 'incidental' PE is to be preferred within this context [1]. It is noteworthy that a small observational cohort study suggested that 'symptomatic' incidental PE might be associated with poorer survival than 'asymptomatic' incidental PE, although this has never been confirmed in other cohort studies [54].

\section{Relevance of incidental PE}

There are several angles from which the relevance of incidental PE could be considered, i.e. comparison of the prognosis of 1) incidental PE in cancer patients versus cancer patients without VTE; 2) incidental PE with a large versus a smaller embolic load; and 3) incidental PE versus symptomatic PE. Most studies in incidental PE have been performed in cancer patients, because they comprise the overall majority of incidental PE diagnoses detected by CT scanning performed for cancer staging.

The relevance of incidental PE could best be demonstrated by showing its natural history without anticoagulant treatment. Unfortunately, there are very few studies to demonstrate this phenomenon. The landmark trial of Barritt and Jordan [55] showed that untreated symptomatic PE is followed by high recurrent $\mathrm{PE}$, and even fatal $\mathrm{PE}$. In fact, of the 19 patients who were not administered anticoagulant treatment, almost 25\% died of fatal PE, and five showed progression of PE at 3 months, whereas none of the treated patients suffered PE-related death or progression [55]. One study in patients with incidental PE evaluated the safety of withholding anticoagulant treatment [56]. Patients with confirmed distal or proximal DVT, but without symptoms of PE randomly received i.v. heparin, followed by vitamin-K antagonists, or a non-steroidal agent, phenylbutazone. A total of 42 out of 90 study patients (47\%) were found to have silent PE. Perfusion-ventilation lung scans showed no significant changes at 10 and 60 days, between the two groups. Moreover, within 12 months, six patients died in the anticoagulant group, with one death being attributed to PE. Furthermore, seven patients died in the non-anticoagulant group, none of which was reportedly related to PE, suggesting that anticoagulant therapy could have been withheld. The small sample size however, does not permit a strong conclusion. Notably, a recent patient level meta-analysis indicated that patients with incidental PE who remained untreated - presumably due to a high risk of bleeding or other relevant prognostic factors- have a very high 6-month risk of recurrent VTE (12\%) and mortality (47\%) [57]. Moreover, data from a retrospective observational study that was not included in the aforementioned meta-analysis confirmed that patients with incidental PE who did not receive anticoagulant treatment showed poorer survival [58].

Several retrospective studies in patients with cancer indicate that incidental PE can have important effects on survival, as well as on recurrent VTE [50, 59-65]. In one study that included a control group of patients without PE, matched for age, cancer histology and severity, the median survival in patients with incidental PE was 8 months, compared to 12 months for the matched controls [50]. In two studies of heterogeneous groups of cancer patients, incidental PE had a similar influence on survival, compared to cancer patients with symptomatic PE. In the first study, 6-month mortality rates were around $50 \%$ in both groups, which was significantly worse than the $27 \%$ mortality rate in the 60 patients with symptoms suggestive of PE, but for whom the diagnosis of PE was excluded [59]. In the second study, 12-month mortality rates were $\sim 53 \%$ in both groups. In addition, a similar incidence of recurrent VTE between patients with symptomatic VTE and patients with incidental $\mathrm{PE}(16.9 \%$ versus $13.3 \%$; $\mathrm{p}=0.77)$ was observed [60]. In contrast, a very recent observational study of a relatively small number of 66 patients reported that malignancy-associated incidental $\mathrm{PE}$ was not associated with a higher risk of mortality, compared to matched control patients without incidental PE [66].

It is well known that in symptomatic PE, a higher thrombus load is associated with greater right ventricular function and increased PE-related mortality [67]. Whether the embolic burden has an impact on prognosis in incidental PE remains an unanswered question. A survey among clinicians showed nonetheless that the more proximal a pulmonary clot is located, the more physicians are willing to treat a patient with anticoagulants [68]. Thus, when a patient is presented with incidental subsegmental PE, 72\% of the clinicians surveyed would initiate anticoagulant treatment in a patient without malignancy, whereas $89 \%$ would treat in the presence of malignancy. If an incidental PE is located in a segmental pulmonary artery, $98 \%$ would treat, irrespective of whether or not a malignancy is present. All would treat when a PE is localised centrally. Another study that evaluated the response of physicians to a diagnosis of incidental PE confirmed these findings with a higher threshold to initiate treatment if the PE was located in a 
subsegmental pulmonary artery [69]. Importantly, in cancer patients receiving ongoing cancer therapy, the continued high risk of recurrent VTE might strongly support a decision to initiate anticoagulation therapy, irrespective of the clot burden. Studies have shown contradictory results, which is partly because incidental and symptomatic subsegmental PE have sometimes been confused. In one study, the presence of an incidental subsegmental PE in 17 patients did not affect survival, compared to age-, histology-, and stage-matched controls [50]. However, when patients with incidental subsegmental PE were compared to those with more proximally located pulmonary clots, the VTE recurrence risk showed no difference [70].

In summary, despite the aforementioned limitations of retrospective studies on incidental PE in cancer patients, there is a general consensus that the prognosis of incidental PE is similar to that of symptomatic PE with regard to the risk of recurrent VTE.

\section{Treatment of incidental PE}

Because incidental PE and symptomatic PE have a similar prognosis, there is a general opinion-based recommendation in several guidelines, including those of the American College of Chest Physicians (Grade 2B) and American Society of Clinical Oncology (informal consensus, moderate strength recommendation), to use the same treatment strategy for patients with incidental DVT and PE and symptomatic VTE patients (table 1) [71, 72]. In particular, patients might have already been discharged, at the time of diagnosis, since cancer staging scans are often viewed in retrospect. Because of the incidental nature of the $\mathrm{PE}$, anticoagulant treatment could be safely started at home, provided the patient receives proper instructions. In contrast to symptomatic PE [73-76], large prospective trials evaluating the safety of outpatient treatment of incidental PE are unavailable, but findings in smaller cohorts indicate that home treatment is not associated with a higher risk of recurrent VTE, bleeding or death [77].

The standard treatment for incidental PE in patients without cancer is direct oral anticoagulants, mostly because of their superior safety profile compared to conventional anticoagulant treatment regimens [70, 71, 78]. Interestingly, the 2016 American College of Chest Physicians guideline suggests no treatment for low-risk patients with symptomatic or asymptomatic isolated subsegmental PE, in cases of normal bilateral ultrasonography of the legs (Grade 2C) [71]. The main argument for this recommendation is that a diagnosis of subsegmental $\mathrm{PE}$ is more likely to be a false-positive finding than a diagnosis of $\mathrm{PE}$ in the segmental or more proximal pulmonary arteries, and 'true' subsegmental PE is likely to have arisen from DVT. Accordingly, the risk of progressive or recurrent VTE without anticoagulation is expected to be lower if concomitant DVT is ruled out. It might be argued that bilateral ultrasonography of the legs could be applied in patients with incidental PE and a high risk of bleeding, since this could help clinicians to better assess the risk/benefit ratio of anticoagulant therapy in the individual patient.

The standard treatment for patients with VTE and cancer is still therapeutic low-molecular weight heparin (LMWH) for a period of 3-6 months [71, 72]. This is because LMWH treatment is associated with less bleeding and lower levels of recurrent VTE compared to vitamin K antagonists $[79,80]$. Patients should be counselled for acceptance of daily injections at regular intervals. A recent study showed that within 6 months, one in five patients had to cease LMWH injections [81]. The most frequent reason for cessation was pain at the injection site. There is uncertainty as to whether treatment should be continued after the

\section{TABLE 1 Overview and practical aspects of management of incidental pulmonary embolism (PE)}

\section{Optimal anticoagulant regime}

Cancer-associated incidental PE

Non-cancer-associated incidental PE

\section{Optimal treatment duration}

Cancer- associated incidental PE

Non-cancer-associated provoked incidental PE

Non-cancer-associated unprovoked incidental PE

Outpatient treatment

All incidental PE
LMWH for the first 6 months

Alternatively: switch to VKA if subcutaneous therapy is not tolerated

DOAC

Alternatively: conventional treatment with VKA if DOAC is contraindicated

At least 6 months and beyond for the duration of active disease

3 months

At least 3 months, indefinite if risk of bleeding is low

In the case of an outpatient, consider initiation of anticoagulant treatment at home, provided that adequate instructions are given to the patient

In the case of an inpatient, initiate anticoagulant therapy in the hospital

LMWH: low-molecular weight heparin; VKA: vitamin K antagonist; DOAC: direct oral anticoagulant. 
initial 6 months. Because of the similar risks as those for symptomatic PE, the current guidelines suggest continuation of anticoagulant treatment if the cancer has not been cured. One cohort study showed that if the cancer is in remission for at least 6 months, the risk of recurrent VTE after cessation of anticoagulant therapy is very low with an incidence of 3.2 per 100 patient-years [82]. Based on this study, it might be reasonable to consider cessation of anticoagulant treatment for cancer-associated VTE once patients have been successfully treated. Importantly, a very recent observational study demonstrated that the risks of major, fatal bleeding during anticoagulant treatment of cancer-associated incidental PE are higher than those of recurrent PE and fatal PE, indicating that anticoagulant therapy comes with inherent risks [83]. It is not known whether recently derived and validated bleeding prediction scores would be applicable and helpful in determining optimal duration of anticoagulation in incidental PE [84-86]. These data call for prospective studies to determine the optimal treatment duration in this patient category.

A recent meta-analysis concluded that both the efficacy and safety of novel oral anticoagulants in the treatment of cancer-associated acute symptomatic VTE are at least comparable with those of vitamin-K antagonists. [87]. However, the event rates were less than those of previous trials in patients with active cancer who were receiving chemotherapy. Importantly, the comparator regime in these studies was LMWH, followed by vitamin-K antagonists. Therefore, LMWH remains the treatment of choice for cancer-associated (incidental) PE. Currently, an international observational study aims to shed more light on the efficacy and safety of current treatment approaches for incidental PE in cancer patients (NCT01727427).

In the absence of any relevant studies in non-cancer associated incidental PE, the aforementioned treatment recommendations might be extrapolated to all patients with incidental PE [1].

\section{Solution to clinical case}

The CT scan was re-evaluated by two senior chest radiologists, who confirmed the presence of subsegmental PE. The decision was made to perform bilateral compression ultrasonography of the legs, which showed no signs of DVT. Considering the high risk of VTE in patients with severe trauma in addition to the current CT findings, and despite the normal compression ultrasonography results, anticoagulation therapy with apixaban was initiated after a chest tube was inserted. The patient recovered completely without bleeding complications and anticoagulant treatment was discontinued after 3 months.

\section{Conclusion}

Incidental PE is a frequent finding on routine CT scans of the chest, with an incidence frequency of $1.1 \%$ in coronary CT and $3.6 \%$ in oncological CT. This prevalence, and in particular the incidence of subsegmental incidental PE, has increased in recent years with advancing CT technology. Large, high quality outcome trials on the optimal management of patients with incidental PE are lacking. It has nonetheless been shown that the finding of intraluminal filling defects in the pulmonary arterial tree on a CT scan performed for purposes other than suspected PE is indicative of PE. Confirmatory diagnostic tests for PE such as CTPA or lung scintigraphy are not indicated, provided the quality of the CT scan is judged as adequate by an experienced radiologist (e.g. no breathing artefacts). The natural course of incidental PE is comparable to that of symptomatic PE with regards to the risk of recurrent venous thrombotic disease and mortality. Because of this observation, guidelines suggest the application of similar management decisions for patients with incidental PE as those with symptomatic PE, including the choice of optimal drug class, outpatient treatment and total duration of treatment.

\section{Acknowledgements}

Frederikus A. Klok and Menno V. Huisman both wrote the manuscript and approved the final version.

\section{References}

1 Di Nisio M, Lee AY, Carrier M, et al. Diagnosis and treatment of incidental venous thromboembolism in cancer patients: guidance from the SSC of the ISTH. J Thromb Haemost 2015; 13: 880-883.

2 Grosse SD, Nelson RE, Nyarko KA, et al. The economic burden of incident venous thromboembolism in the United States: A review of estimated attributable healthcare costs. Thromb Res 2016; 137: 3-10.

3 Liebman HA, O'Connell C. Incidental venous thromboembolic events in cancer patients: what we know in 2016. Thromb Res 2016; 140: Suppl. 1, S18-S20.

4 O'Connell C. How I treat incidental pulmonary embolism. Blood 2015; 125: 1877-1882.

5 Monreal M, Rey-Joly BC, Ruiz MJ, et al. Asymptomatic pulmonary embolism in patients with deep vein thrombosis. Is it useful to take a lung scan to rule out this condition? J Cardiovasc Surg (Torino) 1989; 30: 104-107.

6 Huisman MV, Buller HR, ten Cate JW, et al. Unexpected high prevalence of silent pulmonary embolism in patients with deep venous thrombosis. Chest 1989; 95: 498-502.

7 Dorfman GS, Cronan JJ, Tupper TB, et al. Occult pulmonary embolism: a common occurrence in deep venous thrombosis. AJR Am J Roentgenol 1987; 148: 263-266.

8 Moser KM, Fedullo PF, LitteJohn JK, et al. Frequent asymptomatic pulmonary embolism in patients with deep venous thrombosis. JAMA 1994; 271: 223-225. 
9 Stein PD, Matta F, Musani MH, et al. Silent pulmonary embolism in patients with deep venous thrombosis: a systematic review. Am J Med 2010; 123: 426-431.

10 Boc A, Vene N, Stalc M, et al. Unprovoked proximal venous thrombosis is associated with an increased risk of asymptomatic pulmonary embolism. Thromb Res 2014; 133: 1011-1015.

11 Tzoran I, Saharov G, Brenner B, et al. Silent pulmonary embolism in patients with proximal deep vein thrombosis in the lower limbs. J Thromb Haemost 2012; 10: 564-571.

12 Jimenez D, Diaz G, Marin E, et al. The risk of recurrent venous thromboembolism in patients with unprovoked symptomatic deep vein thrombosis and asymptomatic pulmonary embolism. Thromb Haemost 2006; 95: 562-566.

13 Stein PD, Henry JW. Prevalence of acute pulmonary embolism among patients in a general hospital and at autopsy. Chest 1995; 108: 978-981.

14 Kakkar N, Vasishta RK. Pulmonary embolism in medical patients: an autopsy-based study. Clin Appl Thromb Hemost 2008; 14: 159-167.

15 Sweet PH III, Armstrong T, Chen J, et al. Fatal pulmonary embolism update: 10 years of autopsy experience at an academic medical center. JRSM Short Rep 2013; 4: 2042533313489824.

16 Sandler DA, Martin JF. Autopsy proven pulmonary embolism in hospital patients: are we detecting enough deep vein thrombosis? J R Soc Med 1989; 82: 203-205.

17 Bergqvist D, Lindblad B. A 30-year survey of pulmonary embolism verified at autopsy: an analysis of 1274 surgical patients. Br J Surg 1985; 72: 105-108.

18 Williams JW, Eikman EA, Greenberg S. Asymptomatic pulmonary embolism. A common event in high risk patients. Ann Surg 1982; 195: 323-327.

19 Keenan AM, Palevsky HI, Steinberg ME, et al. An evaluation of preoperative and postoperative ventilation and perfusion lung scintigraphy in the screening for pulmonary embolism after elective orthopedic surgery. Clin Nucl Med 1991; 16: 13-19.

20 Fredin HO, Arborelius M Jr., Hellekant C. Scintigraphy and chest radiography in the screening of pulmonary embolism after total hip replacement. Respiration 1985; 47: 171-176.

21 Machaalany J, Yam Y, Ruddy TD, et al. Potential clinical and economic consequences of noncardiac incidental findings on cardiac computed tomography. J Am Coll Cardiol 2009; 54: 1533-1541.

22 Brenner DJ, Hall EJ. Computed tomography--an increasing source of radiation exposure. N Engl J Med 2007; 357: 2277-2284.

23 Bach AG, Meyer HJ, Taute BM, et al. The frequency of incidental pulmonary embolism in different CT examinations. Br J Radiol 2016; 89: 20150737.

24 Sebastian AJ, Paddon AJ. Clinically unsuspected pulmonary embolism--an important secondary finding in oncology CT. Clin Radiol 2006; 61: 81-85.

25 Cronin CG, Lohan DG, Keane M, et al. Prevalence and significance of asymptomatic venous thromboembolic disease found on oncologic staging CT. AJR Am J Roentgenol 2007; 189: 162-170.

26 Rita LA, Calandriello L, Maggi F, et al. Prevalence of incidental pulmonary emboli in oncology patients. Radiology 2007; 245: 921-922.

27 Browne AM, Cronin CG, English C, et al. Unsuspected pulmonary emboli in oncology patients undergoing routine computed tomography imaging. J Thorac Oncol 2010; 5: 798-803.

28 Tresoldi S, Flor N, Luciani A, et al. Contrast enhanced chest-MDCT in oncologic patients. Prospective evaluation of the prevalence of incidental pulmonary embolism and added value of thin reconstructions. Eur Radiol 2015; 25: 3200-3206.

29 Bach AG, Beckel C, Schurig N, et al. Imaging characteristics and embolus burden of unreported pulmonary embolism in oncologic patients. Clin Imaging 2015; 39: 237-242.

30 Larsen AC, Dabrowski T, Frokjaer JB, et al. Prevalence of venous thromboembolism at diagnosis of upper gastrointestinal cancer. Br J Surg 2014; 101: 246-253.

31 Gladish GW, Choe DH, Marom EM, et al. Incidental pulmonary emboli in oncology patients: prevalence, CT evaluation, and natural history. Radiology 2006; 240: 246-255.

32 Ritchie G, McGurk S, McCreath C, et al. Prospective evaluation of unsuspected pulmonary embolism on contrast enhanced multidetector CT (MDCT) scanning. Thorax 2007; 62: 536-540.

33 Hui GC, Legasto A, Wittram C. The prevalence of symptomatic and coincidental pulmonary embolism on computed tomography. J Comput Assist Tomogr 2008; 32: 783-787.

34 Storto ML, Di CA, Guido F, et al. Incidental detection of pulmonary emboli on routine MDCT of the chest. AJR Am J Roentgenol 2005; 184: 264-267.

35 Winston CB, Wechsler RJ, Salazar AM, et al. Incidental pulmonary emboli detected at helical CT: effect on patient care. Radiology 1996; 201: 23-27.

36 Gosselin MV, Rubin GD, Leung AN, et al. Unsuspected pulmonary embolism: prospective detection on routine helical CT scans. Radiology 1998; 208: 209-215.

37 Engelke C, Rummeny EJ, Marten K. Pulmonary embolism at multi-detector row CT of chest: one-year survival of treated and untreated patients. Radiology 2006; 239: 563-575.

38 Schultz DJ, Brasel KJ, Washington L, et al. Incidence of asymptomatic pulmonary embolism in moderately to severely injured trauma patients. J Trauma 2004; 56: 727-731.

39 Mueller J, Jeudy J, Poston R, et al. Cardiac CT angiography after coronary bypass surgery: prevalence of incidental findings. AJR Am I Roentgenol 2007; 189: 414-419.

40 Haller S, Kaiser C, Buser P, et al. Coronary artery imaging with contrast-enhanced MDCT: extracardiac findings. AJR Am J Roentgenol 2006; 187: 105-110.

41 Wang J, Wu H, Lv B, et al. Asymptomatic pulmonary embolism following off-pump coronary artery bypass: Outcomes of a prospective observational study. Cardiology 2014; 187: Suppl. 1, 79-80.

42 Staskiewicz G, Czekajska-Chehab E, Adamczyk P, et al. Incidental pulmonary embolism identified in cardiac ECG-MDCT studies: Frequency, predisposing factors. Eur Heart J Cardiovasc Imaging 2012; 13: Suppl. 1, P1067.

43 Bach AG, Schmoll HJ, Beckel C, et al. Pulmonary embolism in oncologic patients: frequency and embolus burden of symptomatic and unsuspected events. Acta Radiol 2014; 55: 45-53.

44 Di Nisio M, Ferrante N, De Tursi M, et al. Incidental venous thromboembolism in ambulatory cancer patients receiving chemotherapy. Thromb Haemost 2010; 104: 1049-1054. 
45 Carrier M, Righini M, Wells PS, et al. Subsegmental pulmonary embolism diagnosed by computed tomography: incidence and clinical implications. A systematic review and meta-analysis of the management outcome studies. $J$ Thromb Haemost 2010; 8: 1716-1722.

46 den Exter PL, Kroft LJ, van der Hulle T, et al. Embolic burden of incidental pulmonary embolism diagnosed on routinely performed contrast-enhanced computed tomography imaging in cancer patients. $J$ Thromb Haemost 2013; 11: 1620-1622.

47 Klok FA, van der Bijl N, Eikenboom HC, et al. Comparison of CT assessed right ventricular size and cardiac biomarkers for predicting short-term clinical outcome in normotensive patients suspected of having acute pulmonary embolism. J Thromb Haemost 2010; 8: 853-856.

48 van der Bijl N, Klok FA, Huisman MV, et al. Measurement of right and left ventricular function by ECG-synchronized CT scanning in patients with acute pulmonary embolism: usefulness for predicting short-term outcome. Chest 2011; 140: 1008-1015.

49 den Exter PL, van der Hulle T, Hartmann IJ, et al. Reliability of diagnosing incidental pulmonary embolism in cancer patients. Thromb Res 2015; 136: 531-534.

50 O'Connell C, Razavi P, Ghalichi M, et al. Unsuspected pulmonary emboli adversely impact survival in patients with cancer undergoing routine staging multi-row detector computed tomography scanning. J Thromb Haemost 2011; 9: 305-311.

51 Pena E, Kimpton M, Dennie C, et al. Difference in interpretation of computed tomography pulmonary angiography diagnosis of subsegmental thrombosis in patients with suspected pulmonary embolism. J Thromb Haemost 2012; 10: 496-498.

52 O'Connell CL, Boswell WD, Duddalwar V, et al. Unsuspected pulmonary emboli in cancer patients: clinical correlates and relevance. J Clin Oncol 2006; 24: 4928-4932.

53 Font C, Farrus B, Vidal L, et al. Incidental versus symptomatic venous thrombosis in cancer: a prospective observational study of 340 consecutive patients. Ann Oncol 2011; 22: 2101-2106.

54 O'Connell CL, Razavi PA, Liebman HA. Symptoms adversely impact survival among patients with cancer and unsuspected pulmonary embolism. J Clin Oncol 2011; 29: 4208-4209.

55 Barritt DW, Jordan SC. Anticoagulant drugs in the treatment of pulmonary embolism. A controlled trial. Lancet 1960; 1: 1309-1312.

56 Nielsen HK, Husted SE, Krusell LR, et al. Anticoagulant therapy in deep venous thrombosis. A randomized controlled study. Thromb Res 1994; 73: 215-226.

57 van der Hulle T, den Exter PL, Planquette B, et al. Risk of recurrent venous thromboembolism and major hemorrhage in cancer-associated incidental pulmonary embolism among treated and untreated patients: a pooled analysis of 926 patients. J Thromb Haemost 2016; 14: 105-113.

58 Sun JM, Kim TS, Lee J, et al. Unsuspected pulmonary emboli in lung cancer patients: the impact on survival and the significance of anticoagulation therapy. Lung Cancer 2010; 69: 330-336.

59 Dentali F, Ageno W, Giorgi PM, et al. Prognostic relevance of an asymptomatic venous thromboembolism in patients with cancer. J Thromb Haemost 2011; 9: 1081-1083.

60 den Exter PL, Hooijer J, Dekkers OM, et al. Risk of recurrent venous thromboembolism and mortality in patients with cancer incidentally diagnosed with pulmonary embolism: a comparison with symptomatic patients. J Clin Oncol 2011; 29: 2405-2409.

61 Callejas MF, Errazuriz JI, Castillo F, et al. Incidental venous thromboembolism detected by PET-CT in patients with cancer: prevalence and impact on survival rate. Thromb Res 2014; 133: 750-755.

62 Chaturvedi S, Sidana S, Elson P, et al. Symptomatic and incidental venous thromboembolic disease are both associated with mortality in patients with prostate cancer. PLoS One 2014; 9: e94048.

63 Connolly GC, Menapace L, Safadjou S, et al. Prevalence and clinical significance of incidental and clinically suspected venous thromboembolism in lung cancer patients. Clin Lung Cancer 2013; 14: 713-718.

64 Grigoropoulos NF, Shaw AS, Hampson FA, et al. Incidental pulmonary emboli in lymphoma patients are associated with aggressive disease and poor prognosis. J Thromb Haemost 2010; 8: 2835-2836.

65 Menapace LA, Peterson DR, Berry A, et al. Symptomatic and incidental thromboembolism are both associated with mortality in pancreatic cancer. Thromb Haemost 2011; 106: 371-378.

66 Sahut DM, Caumont PA, Planquette B, et al. Risk factors and clinical outcome of unsuspected pulmonary embolism in cancer patients: a case-control study. J Thromb Haemost 2012; 10: 2032-2038.

67 Meinel FG, Nance JW Jr., Schoepf UJ, et al. Predictive value of computed tomography in acute pulmonary embolism: systematic review and meta-analysis. Am J Med 2015; 128: 747-759.

68 den Exter PL, van Roosmalen MJ, van den Hoven P, et al. Physicians' management approach to an incidental pulmonary embolism: an international survey. J Thromb Haemost 2013; 11: 208-213.

69 Franklin JM, Rahman N, Gleeson FV. The clinician's response to a report of an incidental pulmonary embolism detected on multidetector CT. Postgrad Med J 2011; 87: 746-749.

70 van der Hulle T, Kooiman J, den Exter PL, et al. Effectiveness and safety of novel oral anticoagulants as compared with vitamin $\mathrm{K}$ antagonists in the treatment of acute symptomatic venous thromboembolism: a systematic review and meta-analysis. J Thromb Haemost 2014; 12: 320-328

71 Kearon C, Akl EA, Ornelas J, et al. Antithrombotic therapy for VTE disease: CHEST Guideline and Expert Panel Report. Chest 2016; 149: 315-352.

72 Lyman GH, Khorana AA, Kuderer NM, et al. Venous thromboembolism prophylaxis and treatment in patients with cancer: American Society of Clinical Oncology clinical practice guideline update. J Clin Oncol 2013; 31: 2189-2204.

73 den Exter PL, Zondag W, Klok FA, et al. Efficacy and safety of outpatient treatment based on the Hestia Clinical Decision Rule with or without N-terminal pro-brain natriuretic peptide testing in patients with acute pulmonary embolism. A randomized clinical trial. Am J Respir Crit Care Med 2016; 194: 998-1006.

74 van der Hulle T, Dronkers CE, Klok FA, et al. Recent developments in the diagnosis and treatment of pulmonary embolism. J Intern Med 2016; 279: 16-29.

75 Zondag W, Kooiman J, Klok FA, et al. Outpatient versus inpatient treatment in patients with pulmonary embolism: a meta-analysis. Eur Respir J 2013; 42: 134-144. 
76 Aujesky D, Roy PM, Verschuren F, et al. Outpatient versus inpatient treatment for patients with acute pulmonary embolism: an international, open-label, randomised, non-inferiority trial. Lancet 2011; 378: 41-48.

77 Font C, Carmona-Bayonas A, Fernández-Martinez A, et al. Outpatient management of pulmonary embolism in cancer: data on a prospective cohort of 138 consecutive patients. J Natl Compr Canc Netw 2014; 12: 365-373.

78 Konstantinides SV, Torbicki A, Agnelli G, et al. 2014 ESC guidelines on the diagnosis and management of acute pulmonary embolism. Eur Heart J 2014; 35: 3033-3069.

79 Lee AY, Kamphuisen PW, Meyer G, et al. Tinzaparin vs warfarin for treatment of acute venous thromboembolism in patients with active cancer: a randomized clinical trial. JAMA 2015; 314: 677-686.

80 Lee AY, Levine MN, Baker RI, et al. Low-molecular-weight heparin versus a coumarin for the prevention of recurrent venous thromboembolism in patients with cancer. N Engl J Med 2003; 349: 146-153.

81 van der Wall SJ, Klok FA, den Exter PL, et al. Continuation of LMWH treatment for cancer related venous thromboembolism - a prospective cohort study in daily clinical practice. J Thromb Haemost 2017; 15: 74-79.

82 van der Hulle T, den Exter PL, van den Hoven P, et al. Cohort study on the management of cancer-associated venous thromboembolism aimed at the safety of stopping anticoagulant therapy in patients cured of cancer. Chest 2016; 149: 1245-1251.

83 Peris M, Jimenez D, Maestre A, et al. Outcome during and after anticoagulant therapy in cancer patients with incidentally found pulmonary embolism. Eur Respir J 2016; 48: 1360-1368.

84 Klok FA, Barco S, Konstantinides SV. External validation of the VTE-BLEED score for predicting major bleeding in stable anticoagulated patients with venous thromboembolism. Thromb Haemost 2017; 117: 1164-1170.

85 Klok FA, Hosel V, Clemens A, et al. Prediction of bleeding events in patients with venous thromboembolism on stable anticoagulation treatment. Eur Respir J 2016; 48: 1369-1376.

86 Klok FA, Kooiman J, Huisman MV, et al. Predicting anticoagulant-related bleeding in patients with venous thromboembolism: a clinically oriented review. Eur Respir J 2015; 45: 201-210.

87 van der Hulle T, den Exter PL, Kooiman J, et al. Meta-analysis of the efficacy and safety of new oral anticoagulants in patients with cancer-associated acute venous thromboembolism. J Thromb Haemost 2014; 12: $1116-1120$. 